\title{
Choice of transcatheter heart valve: should we select the device according to each patient's characteristics or should it be "one valve fits all"?
}

\author{
Matthias Renker ${ }^{1,2}$, Won-Keun Kim ${ }^{1,2,3}$ \\ ${ }^{1}$ Department of Cardiology, ${ }^{2}$ Department of Cardiac Surgery, Kerckhoff Heart Center, Bad Nauheim, Germany; ${ }^{3}$ Department of Cardiology, \\ University of Giessen, Giessen, Germany \\ Contributions: (I) Conception and design: All authors; (II) Administrative support: All authors; (III) Provision of study materials or patients: None; \\ (IV) Collection and assembly of data: All authors; (V) Data analysis and interpretation: All authors; (VI) Manuscript writing: All authors; (VII) Final \\ approval of manuscript: All authors. \\ Correspondence to: Won-Keun Kim. Department of Cardiology, Kerckhoff Heart Center, 61231 Bad Nauheim, Germany. \\ Email: w.kim@kerckhoff-klinik.de.
}

\begin{abstract}
Since its introduction at the beginning of the century, transcatheter aortic valve replacement (TAVR) has implicated a paradigm shift in the treatment of patients with symptomatic aortic valve stenosis. The past years have brought about major improvements of procedural outcomes owing to advances in imaging and patient selection, global experience, and device technology. Whereas in the early stages of TAVR, only two different devices with limited sizes and access options were used, currently a variety of different transcatheter heart valves (THVs) are available. This has expanded the spectrum of patients that can be treated with TAVR and has allowed for sophisticated device selection tailored to the patients' individual anatomy and comorbidities. The big question is whether such a customized device selection is really necessary-or is there one valve type that fits all patients? With this question in mind, the authors provide an overview of contemporary THVs, including technical specifications and clinical data, that help us to understand the potential value of a differential use of THVs.
\end{abstract}

Keywords: Transcatheter aortic valve implantation (TAVI); transcatheter aortic valve replacement (TAVR); transcatheter heart valve (THV); self-expanding; balloon-expandable; mechanically expandable

Submitted Feb 26, 2020. Accepted for publication Mar 13, 2020.

doi: $10.21037 / \mathrm{atm} .2020 .04 .13$

View this article at: http://dx.doi.org/10.21037/atm.2020.04.13

\section{Introduction}

Transcatheter aortic valve replacement (TAVR) has become the standard therapy for patients with severe aortic stenosis and high or prohibitive surgical risk $(1,2)$. Furthermore, robust data showing favorable results in comparison to surgical aortic valve repair have led to its adoption in intermediate risk patients (3-5). Recently, two landmark trials have demonstrated non-inferiority and even superiority of transfemoral TAVR in low-risk patients $(6,7)$. Increased utilization of this treatment rests upon major advances in terms of knowledge, careful patient selection, sophisticated imaging, and evolving novel technologies.
Issues that dominated the early period of TAVR were frequent procedural complications, paravalvular leakage (PVL), stroke, conduction disturbances requiring permanent pacemaker implantation (PPI), access related complications, renal failure, sequelae of false sizing including device embolization or annular rupture, and coronary occlusion. The first generation of transcatheter heart valves (THV), represented by the balloon-expandable (b-exp) Edwards Sapien and the self-expanding (s-exp) Medtronic CoreValve, was characterized by large-bore delivery systems and limited availability of annulus sizes. During recent years, iterations of existing THVs as well as devices with novel concepts 

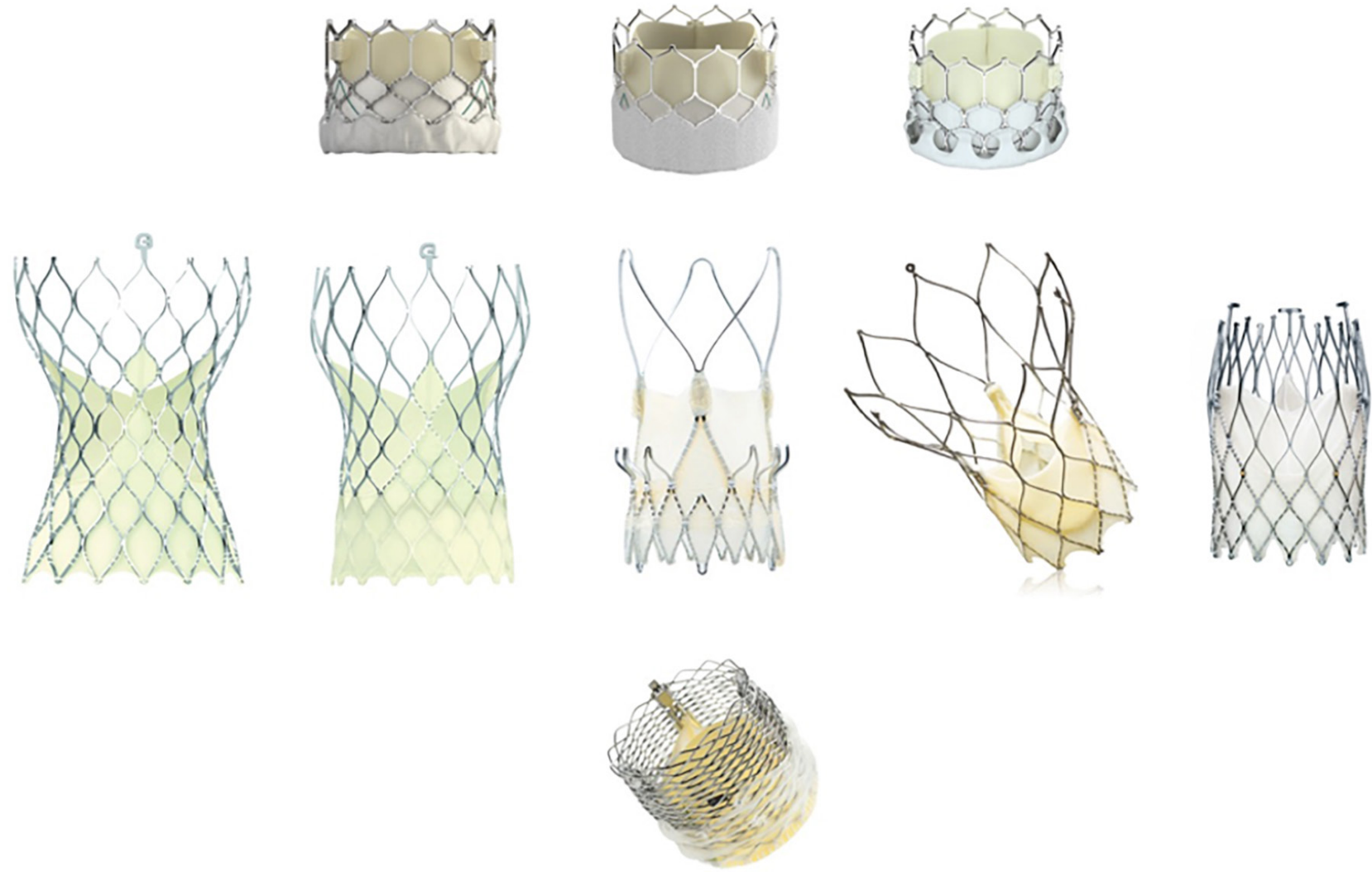

Figure 1 Currently available THVs with approval for the European and US market. Upper row from left to right: balloon-expandable prostheses SAPIEN 3 (Edwards Lifesciences), SAPIEN 3 Ultra (Edwards Lifesciences), and MyVal (Meril Life Sciences). Middle row from left to right: self-expanding prostheses Evolut R (Medtronic), Evolut PRO (Medtronic), ACURATE neo (Boston Scientific), Portico (Abbott Vascular), and ALLEGRA (New Valve Technology). Lower row: mechanically expandable prosthesis LOTUS Edge (Boston Scientific). Image source: each manufacturer.

have provided substantial improvements that overcome the limitations of the early-generation devices. This has increased the ease of use, lowered the rate of complications, and ameliorated patient outcomes. Hence, treatment of a broader spectrum of patients has become feasible and sophisticated selection of the appropriate THV tailored to patients' individual anatomy and comorbidities is now possible. The purpose of the present article is to provide an overview of contemporary THVs and their technical specifications and review clinical data, placing an emphasis on differential use in specific clinical scenarios.

\section{Overview of currently available THVs}

All currently commercially available THVs are displayed in Figure 1 and briefly characterized in Table 1. Specification of existing evidence from clinical trials is presented in Table 2.

\section{Balloon-expandable devices}

\section{SAPIEN 3 and SAPIEN 3 Ultra}

* Access: transvascular, transaortic, transapical;

* Sheath: 14-16 Fr inner diameter;

* Deployment: balloon expansion, mandatory rapid pacing;

* Sizes (annulus range): 20, 23, 26, 29 mm (18.6$29.5 \mathrm{~mm})$.

Originating from the THV used for the first-in-man implantation in 2002 by Cribier (28), the SAPIEN 3 and SAPIEN 3 Ultra (Edwards Lifesciences, Irvine, CA, USA) now constitute the fourth and fifth generation of b-exp devices. Several iterations have contributed to better results 
Table 1 Overview of commercially available transcatheter heart valves

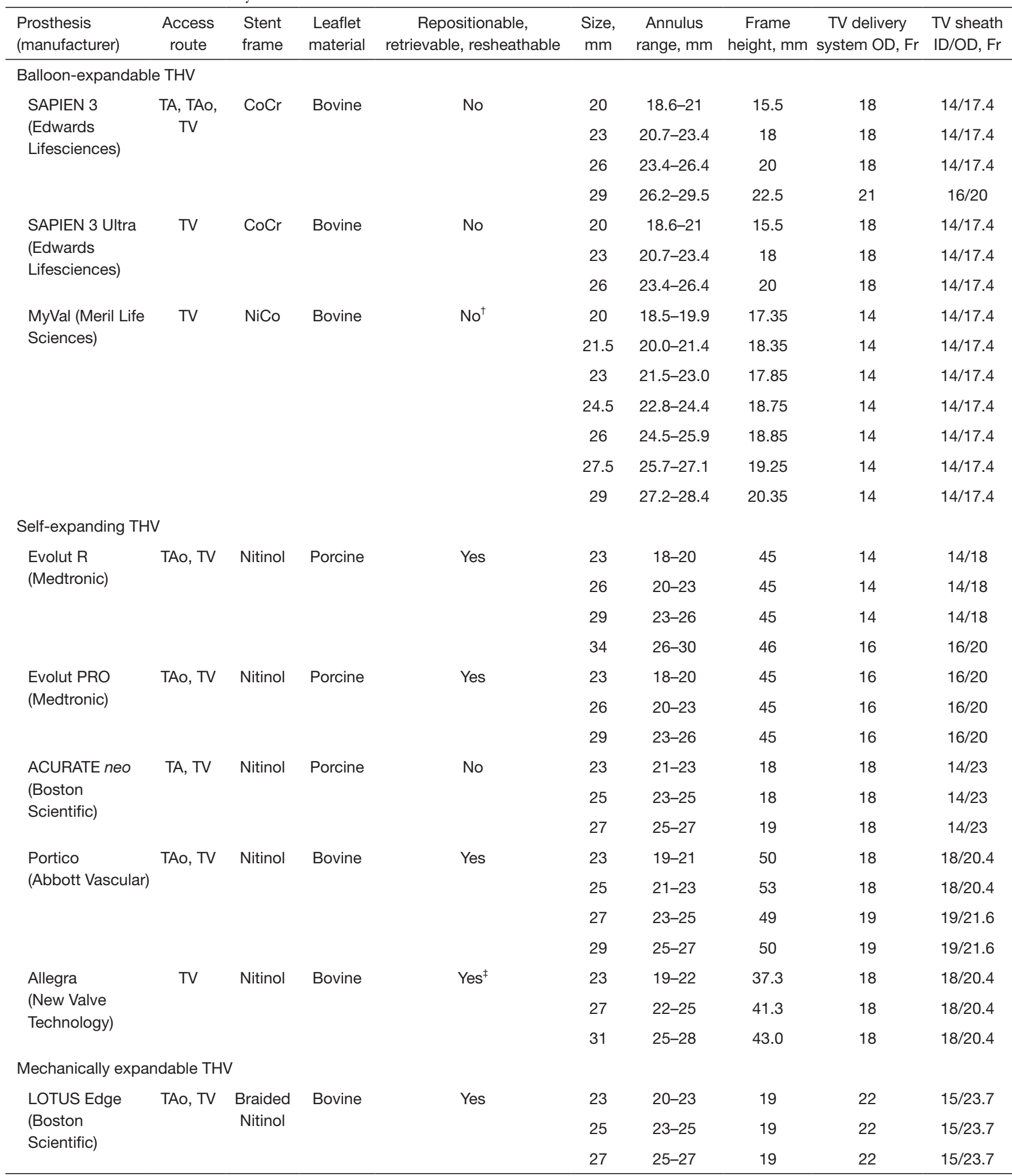

${ }^{\dagger}$, undeployed THV may be fully retrieved from the expandable sheath in case the THV fails to cross the annulus; ${ }^{\ddagger}$, resheathable and repositionable in Permaflow, retrievable until distal valve release. CoChr, Cobalt-Chromium; ID, inner diameter; NiCo, Nickel-Cobalt; OD, outer diameter; TA, transapical; TAo, transaortic; TV, transvascular. 
Table 2 Clinical data of transcatheter heart valves

\begin{tabular}{|c|c|c|c|c|c|c|c|c|}
\hline Manufacturer prosthesis - study & $\begin{array}{c}\text { Patients, } \\
\mathrm{n}\end{array}$ & Operative risk & $\begin{array}{c}\text { 30-day } \\
\text { mortality, \% }\end{array}$ & $\begin{array}{c}\mathrm{PVL} \geq 2^{\circ}, \\
\%\end{array}$ & $\begin{array}{l}\text { Pmean, } \\
\mathrm{mmHg}\end{array}$ & $\begin{array}{c}\text { PPI, } \\
\%\end{array}$ & $\begin{array}{c}\text { Major } \\
\text { vasc, \% }\end{array}$ & $\begin{array}{c}\text { Major } \\
\text { stroke, \% }\end{array}$ \\
\hline \multicolumn{9}{|l|}{ Edwards Lifesciences SAPIEN 3} \\
\hline Webb et al. (CE) (8) & 150 & High (STS 7.4\%) & 4.7 & 3.5 & $10.6 \pm 4.7$ & 13.3 & 5.3 & 0 \\
\hline Kodali et al. (Partner 2 S3 HR) (9) & 583 & High (STS 8.7\%) & 2.2 & 3.7 & $11.4 \pm 4.8$ & 13 & 5 & 0.9 \\
\hline \multicolumn{9}{|l|}{ Edwards Lifesciences SAPIEN 3 Ultra } \\
\hline Saia et al. (S3U) (11) & 139 & Intermed (STS 3.8\%) & 0 & 1.4 & $11.6 \pm 4.3^{\dagger}$ & 4.4 & 2.2 & 0 \\
\hline \multicolumn{9}{|l|}{ Medtronic Evolut R } \\
\hline \multicolumn{9}{|l|}{ Medtronic Evolut PRO } \\
\hline Forrest et al. (US Clinical Study) (14) & 60 & Intermed (STS 6.4\%) & 1.7 & 0 & $6.4 \pm 2.1$ & 11.8 & 10 & 1.7 \\
\hline \multicolumn{9}{|l|}{ Boston Scientific ACURATE neo } \\
\hline Möllmann et al. (CE) (15) & 89 & High (ES I 26.5\%) & 3.4 & 4.9 & $8.0 \pm 2.9$ & 8 & 3.4 & 2.2 \\
\hline Kim et al. (SAVI TF) (16) & 1,000 & Intermed (STS 6.0\%) & 1.3 & $4.1^{\S}$ & $8.3 \pm 4.0^{\S}$ & 8.2 & 3.8 & 1.9 \\
\hline \multicolumn{9}{|l|}{ Boston Scientific LOTUS/LOTUS Edge } \\
\hline Meredith et al. (REPRISE II/CE) (17) & 120 & Intermed (STS 7.1\%) & 4.2 & 1 & $11.5 \pm 5.2$ & 28.6 & 0 & 1.7 \\
\hline \multicolumn{9}{|l|}{ Abbott Vascular Portico } \\
\hline Manoharan et al. (CE) (22) & 102 & Intermed (STS 5.6\%) & 2.9 & 3.8 & $8.9 \pm 3.8$ & 9.8 & 5.9 & 2.9 \\
\hline Maisano et al. (Portico-1) (23) & 941 & Intermed (STS 5.8\%) & 2.7 & 3.9 & $8.6 \pm 3.9$ & 18.7 & 5.5 & 1.6 \\
\hline Fontana et al. (PORTICO IDE) (24) & 381 & Intermed (STS 6.4\%) & 3.5 & 6.3 & 8.4 & 27.7 & 9.6 & 1.6 \\
\hline \multicolumn{9}{|l|}{ New Valve Technology ALLEGRA } \\
\hline Wenaweser et al. (Allegra FIM) (25) & 21 & High (ES I $30.4 \%$ ) & 4.8 & 5.3 & $8.9 \pm 3$ & 23.8 & 14.3 & 0 \\
\hline Jagielak et al. (Allegra Pilot) (26) & 27 & Intermed (ES I 12.4\%) & 0 & 17.4 & 9 & 8 & 0 & 0 \\
\hline \multicolumn{9}{|l|}{ Meril Life Sciences MyVal } \\
\hline Sharma et al. (FIM/CE) (27) & 30 & Intermed (STS 6.4\%) & 3.3 & 0 & $8.8 \pm 2.5$ & 0 & 6.7 & 0 \\
\hline
\end{tabular}

Data presented as mean \pm standard deviation or median [interquartile range]. ${ }^{\dagger}$, at discharge; ${ }^{\ddagger}$, CoreValve $\mathrm{n}=26$, Evolut $\mathrm{R} n=537$, Evolut PRO $n=162 ;$; , at 7 days post TAVR. ES I, Logistic EuroScore; STS, Society of Thoracic Surgeons Risk Score for Mortality; PVL, paravalvular leak; PPI, permanent pacemaker implantation post TAVR; Major vasc, major vascular complication; TAVR, transcatheter aortic valve replacement. 
by optimizing paravalvular sealing, decreasing diameters of the delivery system, and facilitating a straight-forward procedural flow. The stent frame is made of a cobaltchromium alloy, and the three leaflets consist of bovine pericardium and are attached slightly above the inflow portion, which in the case of the SAPIEN 3 is covered by an internal polyethylene terephthalate (PET) skirt and an additional outer PET cuff to reduce paravalvular regurgitation. To further enhance the sealing mechanism, the SAPIEN 3 Ultra features a textured outer portion of the PET material that has a greater height than that of the SAPIEN 3. Even though pre-dilatation is recommended prior to THV implantation according to the instructions for use, direct implantation without pre-dilatation has become very common. Rapid ventricular pacing during implantation is mandatory, which may be unfavorable in patients with reduced left ventricular function or myocardial ischemia. The positioning of the SAPIEN 3 requires a co-planar view of the annular plane, and due to the radiopaque marker, its placement is very intuitive and can be accomplished in a precise fashion, even in the presence of a horizontal ascending aorta. A slightly higher position has been shown to decrease the risk of conduction disturbances, but in turn may increase the risk of coronary obstruction or impair coronary re-access (29). The SAPIEN 3 and SAPIEN 3 Ultra are characterized by a very effective sealing mechanism that can compensate for suboptimal positioning. The principle of balloon expansion bears the risk of annular rupture, especially in unfavorable calcification patterns and/or undue oversizing. Hence, less oversizing or use of stepwise inflation might be strategies to reduce the risk of annular damage (30). For the SAPIEN 3 and SAPIEN 3 Ultra devices, it is of utmost importance to verify the correct direction of loading on the delivery system, and for femoral access it is vital to retrieve the pusher prior to deployment; otherwise, there is a risk of malpositioning due to displaced balloon position.

\section{MyVal}

* Access: transvascular;

* Sheath: 14 Fr inner diameter;

* Deployment: balloon expansion, mandatory rapid pacing;

* Sizes (annulus range): 20, 21.5, 23, 24.5, 26, 27.5, $29 \mathrm{~mm}(18.5-28.4 \mathrm{~mm})$.

The MyVal (Meril Life Sciences, Vapi, Gujarat, India) is a b-exp tri-leaflet, bovine pericardium THV with an inner and outer sealing mechanism. One of the few differences in comparison with the SAPIEN 3 and SAPIEN 3 Ultra is the stent frame, which is made of nickel-cobalt and has a complete honeycomb design. Thus, under fluoroscopy, the crimped valve therefore has a banding pattern to facilitate positioning. Pre-dilatation is recommended with this THV. Compared with other THVs, the MyVal is available in intermediate sizes with $1.5 \mathrm{~mm}$ increments. This may facilitate a precise size selection with minimized over- or under-sizing. The expandable sheath (14 Fr inner diameter) is compatible with all available THV sizes. However, the experience with this THV is thus far very limited. The Conformité Européenne (CE) mark has only recently been granted based on the first results from the MyVal-1 study (27).

\section{Self-expanding devices}

\section{Evolut R and PRO}

* Access: transvascular, transaortic;

* Sheath: inline sheath with 14 Fr outer diameter equivalent (Evolut R 34 and PRO: 16 Fr);

* Deployment: no rapid pacing, repositionable, resheathable, recapturable;

- Sizes (annulus range): 23, 26, 29, $34 \mathrm{~mm}$; PRO: only 23, 26, $29 \mathrm{~mm}(18-30 \mathrm{~mm})$.

The CoreValve (Medtronic, Minneapolis, MN, USA) was the first s-exp device that was approved for European and US markets. It was afflicted with several shortcomings, including a relatively high rate of PVL and the need for PPI as well as difficult positioning of the THV system. The Evolut R and Evolut PRO are iterations of this platform that were developed to address these issues. Both THV models consist of a nitinol-based stent frame and trileaflet porcine pericardium that is mounted in a supraannular position. The valve design accounts for the low gradients and increased procedural safety and may facilitate optimal positioning (31). The latter may allow for higher positioning, which contributes to lower PPI rates (32). Refinements include improved sealing, especially the addition of a sealing skirt in the PRO model that has led to decreased PVL rates (14). Pre-dilatation is recommended, but given the relatively high radial force, it is not a prerequisite in all cases. The Evolut PRO has a slightly larger delivery system. Together with the SAPIEN 3, the Evolut R is the only THV that has been granted US market approval for application in low-risk patients.

\section{ACURATE neo}

* Access: transvascular, transapical; 
* Sheath: 14 Fr inner diameter;

* Deployment: 2-step, top-down deployment, rapid pacing recommended per instructions for use, but in clinical practice rarely used;

* Sizes (annulus range): 23, 25, $27 \mathrm{~mm}$ (21-27 mm).

The ACURATE neo (Boston Scientific, Marlborough, MA, USA) is a supra-annular, nitinol-based s-exp device with an alloy stent frame that consists of a lower crown in the inflow aspect and an upper crown in the outflow aspect with three stabilization arches. It represents the iteration of the intra-annular ACURATE TA and can be used via transvascular and transapical access. Three porcine pericardium leaflets treated with an anti-calcification process are attached at the waist of the stent, with each commissure aligned with one of three struts at the origin of the stabilization arches. Additionally, the inflow aspect is covered by a porcine pericardium fabric skirt for the purpose of paravalvular sealing. In contrast to all other nitinol-based devices, the ACURATE neo is characterized by a "top-down" deployment which consists of two steps. However, it cannot be resheathed or retrieved. Hemodynamic stability is maintained during the entire deployment.

Further modifications include a delivery system that is compatible with a $14 \mathrm{Fr}$ inner sheath diameter, which required changes in the architecture of the stent. Given the relatively straightforward two-step mechanism of deployment in two steps and intuitive handling, the learning curve is steep and the ease of use contributes to procedural safety. The ACURATE neo device is suitable for most aortic root anatomies, but especially for patients with short coronary distance and horizontal configuration of the ascending aorta. The comparably low radial force makes a sufficient pre-dilatation mandatory, and may be a disadvantage in more calcified annuli with eccentric distribution (33). However, the low radial force also accounts for one of the lowest PPI rates among contemporary devices (34).

\section{Portico}

* Access: transvascular, transaortic;

* Sheath: 18/19 Fr inner diameter;

* Deployment: no rapid pacing, repositionable, resheathable, recapturable;

* Sizes (annulus range): 23, 25, 27, 29 mm (19-27 mm).

The Portico (Abbott Vascular, Santa Clara, CA, USA) is a tri-leaflet s-exp THV consisting of a nitinol stent frame with bovine pericardial leaflets and large stent cells for facilitated coronary access. The inflow aspect is covered by a porcine pericardial sealing cuff, and the outflow aspect incorporates three retention tabs that are attached to the retainer receptacle of the 18 or 19 Fr delivery system. The handle features a rotating deployment wheel for unsheathing the device with a release lever at $80 \%$ release that has to be turned before full deployment is possible. The delivery system is flexible and allows resheathing and repositioning. Furthermore, it is retrievable if needed. As the full radial force of the Portico is reached upon complete stent expansion, pre-dilatation is recommended and should not be omitted as long as there are no data on the feasibility and safety of direct implantation. Rapid pacing is not necessary for the deployment, but fast ventricular pacing might facilitate positioning, particularly in the case of extrasystoles or uncontrolled motion. During deployment, outflow obstruction may occur but usually with only mild to moderate hemodynamic compromise, as the intra-annular leaflet position allows an early valve function that begins at $50 \%$ of deployment.

\section{ALLEGRA}

* Access: transfemoral;

* Sheath: inline sheath with 15 Fr inner diameter, 18 Fr valve cartridge;

* Deployment: no rapid pacing, 3 steps, recapturable;

* Sizes (annulus range): 23, 27, $31 \mathrm{~mm}$ (19-28 mm).

The ALLEGRA (New Valve Technology, Hechingen, Germany) is a supra-annular self-expanding THV with a bovine pericardial tri-leaflet design attached to a nitinol stent frame. The frame is equipped with 6 gold markers that indicate the new valve plane. All valve sizes are delivered via a flexible transfemoral delivery system with an integrated sheath intended to provide stable implantation with reduced friction. The delivery system incorporates the technology for a three-step release intended for an occlusion-free implantation. Therefore, there is no need for fast or rapid pacing during the implantation process. Data on this THV are limited and includes the first-in-human clinical studies showing results with a hemodynamic performance comparable to that of other THVs $(25,26)$. Due to its hemodynamic properties, a potential use of this THV in valve-in-valve settings has been described (35).

\section{Mechanically expandable THV}

\section{LOTUS Edge}

* Access: transvascular, transaortic; 
* Sheath: 21 Fr inner diameter;

* Deployment: no rapid pacing, repositionable, resheathable, recapturable;

* Sizes (annulus range): 23, 25, $27 \mathrm{~mm}$ (20-27 mm).

The LOTUS Edge (Boston Scientific, Marlborough, MA, USA) is a pre-attached mechanically expanding device consisting of bovine pericardial leaflets mounted within a braided nitinol mesh and an outer adaptive seal to minimize PVL. The prosthesis can be deployed stepwise with longitudinal foreshortening that builds up radial force and leads to controlled, mechanical expansion. The intraannular leaflets are fully functional at an early stage of the expansion. Once the position is satisfactory, the stent frame can be locked with a post-and-buckle locking mechanism. After final functional evaluation, the prosthesis can be fully released or repositioned if necessary, making it unique among resheathable devices. The LOTUS Edge is the second generation of the LOTUS valve system with modifications in device design and handling of the delivery system. The so-called "depth-guard technology" facilitates early device anchoring in order to limit the depth of implantation for less conduction disturbances. The LOTUS Edge feasibility study demonstrated a lower rate of new PPI with 15.2\% (20). Prior balloon valvuloplasty was part of the protocol in the LOTUS CE mark study but is rarely used in daily practice (17). The mechanical principle of stent expansion and the outer adaptive seal ensure "surgical-like" results with regard to PVL (0\% relevant PVL in the LOTUS Edge study, and similarly low rates in the studies with the LOTUS valve system) (19,20). Hence, this valve system seems to be favorable for patients with a bicuspid aortic valve anatomy or severe aortic valve calcification. To minimize the risk of PPI, a relatively high positioning may be desirable. However, this might impair re-access to the coronary arteries, particularly in the case of low coronary uptake.

\section{Which device is suitable for which patient?}

A differential selection process requires a thorough evaluation of the patient's individual situation, comorbidities, anatomy of the aortic root, and the potential access route. For procedural planning prior to TAVR, comprehensive imaging with multidetector computed tomography of the aorta and ilio-femoral arteries is mandatory, since it provides details on all aspects required for decision-making. The following criteria should be considered for a customized choice of the THV system (Table 3).

\section{Aortic root anatomy}

\section{Annulus size}

Contemporary THVs are commonly available in 3 or 4 different sizes covering annulus diameters between 18 and $30 \mathrm{~mm}$ (details are provided in Table 1). Most devices are suitable for a maximum annulus size of $27 \mathrm{~mm}$. In the case of larger dimensions, the only options are either the SAPIEN 3 $29 \mathrm{~mm}$ (up to $29.5 \mathrm{~mm}$ ) or the Evolut R $34 \mathrm{~mm}$ (maximum annulus $30 \mathrm{~mm}$ ). In this context, Sathananthan et al. examined overexpansion of the b-exp SAPIEN 3 in an $e x$ vivo setting and concluded that it is feasible, but suggested that excessive overexpansion may increase the risk of acute leaflet failure, impaired function, and reduced durability (26). Good results for overexpansion of the SAPIEN 3 up to annulus sizes of $31 \mathrm{~mm}$ and more were reported in a case series (37). Small annuli bear the risk of high postprocedural gradients and patient-prosthesis mismatch, which is more frequent for intra-annular devices (38). Thus, the use of THVs with supra-annular leaflet attachment may be advantageous in the case of small aortic root dimensions.

In patients with annulus dimensions that are borderline between two prosthesis sizes, the degree of oversizing should also be considered. Whereas for s-exp prostheses a higher degree of oversizing is preferred, for b-exp devices less oversizing may be the better choice. For instance, a patient with an area-derived annulus diameter of $23.6 \mathrm{~mm}$ would be in the lower range of sizing for most s-exp devices (Evolut R 29 mm, ACURATE neo $25 \mathrm{~mm}$, Portico $27 \mathrm{~mm}$ ) with a more favorable extent of oversizing than for the SAPIEN $326 \mathrm{~mm}$, which would have a higher risk of annulus rupture. The risk of aortic annulus rupture is reported to range between $0.5 \%$ and $1 \%$ among TAVR procedures, implying adverse outcomes $(30,39,40)$. For the prosthesis choice, it should be kept in mind that this complication is traditionally more often associated with b-exp than with s-exp devices (30). However, when postdilatation is performed with an oversized balloon, the risk of annulus rupture was suggested to increase with s-exp THVs as well (41).

\section{Coronary distance}

Coronary obstruction is a rare but fulminant complication with high mortality (42). Careful pre-procedural imaging can help to identify anatomical risk factors, including a short distance between the annulus and the coronary ostia and a shallow sinus of Valsalva. Non-anatomical risk factors are higher age, female sex, no previous coronary artery 
Table 3 Valve selection

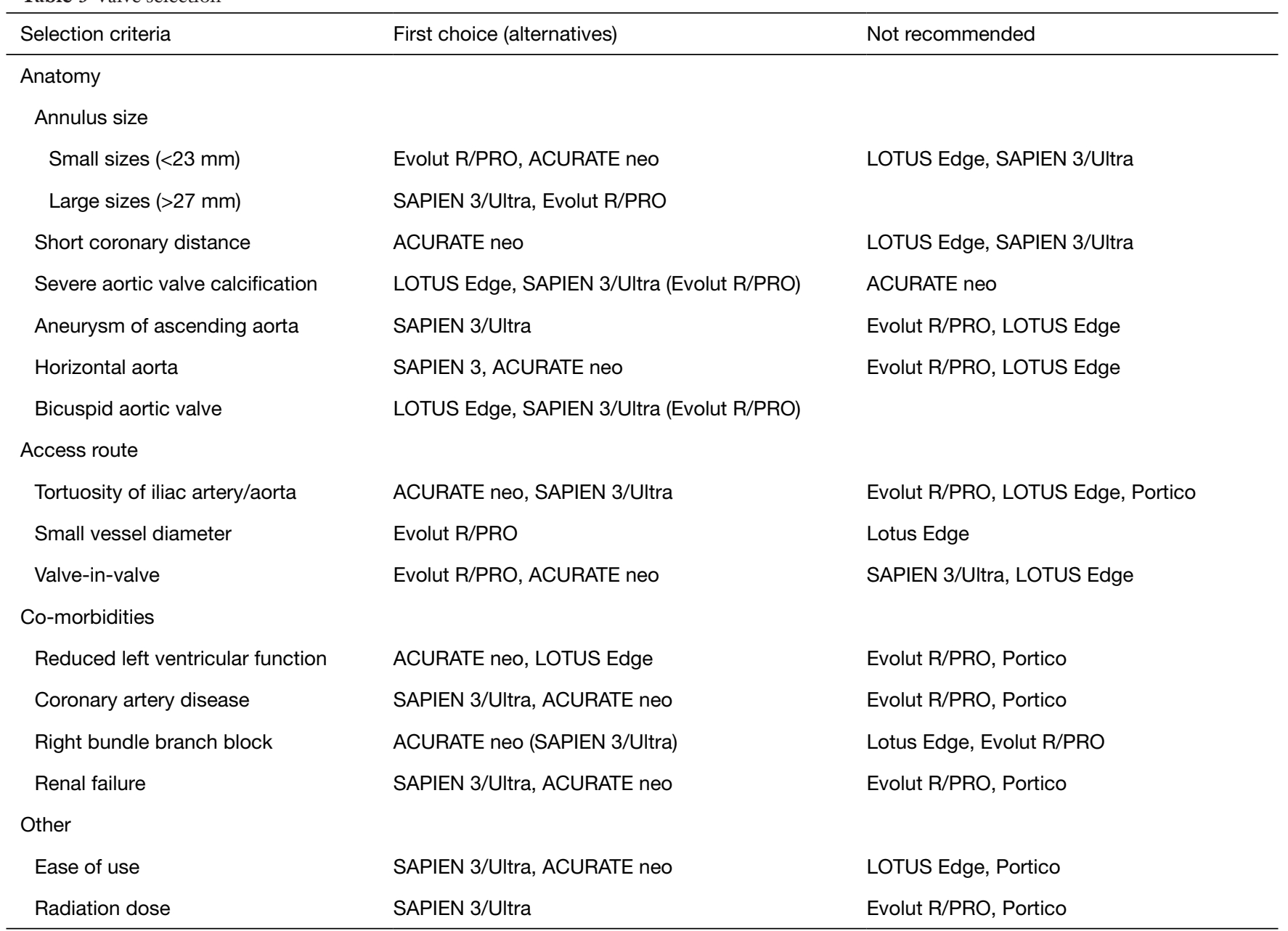

bypass graft, implantation of b-exp valves, and valve-invalve TAVR for failed surgical aortic bioprostheses (43). A lower risk of coronary obstruction may be anticipated for s-exp devices with specific principles of anchoring, for instance the upper crown of the ACURATE neo that keeps the native leaflets away from the coronary ostia, or THVs that can be repositioned or retrieved prior to final deployment.

\section{Calcification}

In contrast to surgical aortic valve replacement, the amount and distribution pattern of aortic valve calcification have a higher impact on outcome for TAVR patients. This has been demonstrated for s-exp as well as for b-exp devices, indicating a higher risk of procedural complications, need for post-dilatation, and PVL (44). However, if there is evidence of severe asymmetric calcification protruding into the left ventricular outflow tract, the selection of a valve with an advanced external sealing skirt may be preferable. As extensive calcification is an independent factor associated with annulus rupture, TAVR with b-exp devices as well as balloon-dilatation in s-exp devices need to be used with caution in this clinical scenario. To date, there is no randomized comparison of different THVs according to degree and distribution of aortic valve calcification. Until reliable evidence on currently available THVs is available, the decision on the appropriate prosthesis to be used for specific degrees and patterns of calcification depends on the individual experience and preference of the operator.

\section{Bicuspid valve morphology}

In comparison with tricuspid aortic valves, bicuspid morphologies are frequently associated with larger annulus dimensions, extensive and complex calcification patterns, 
an asymmetric valve orifice, and a dilated aortic root and ascending aorta $(45,46)$. Therefore, replacement of bicuspid aortic valves via TAVR is challenging, and bicuspid aortic valves are classified as a relative contraindication for TAVR in current guidelines $(1,2)$. Existing data on TAVR in this specific anatomy are not consistent and do not allow to draw firm conclusions. Nevertheless, available registry-based analyses indicate that TAVR is feasible and safe, particular with the use of new-generation devices (46-49). Further research in terms of differential device selection and longterm durability is required, especially because bicuspid anatomies are encountered more frequently in younger patients who are increasingly being referred for TAVR.

\section{Aortic aneurysm/horizontal aorta}

The implantation in a horizontal configuration of the ascending aorta is regarded as challenging due to difficult positioning, especially in s-exp devices with long stent frames. Data on the impact of a horizontal aorta on procedural outcomes, however, are scarce. Nonetheless, it can be assumed that THVs with short stent frames are the most appropriate choice for such anatomies. The b-exp devices feature delivery systems with dual articulation and distal flexing that allows for facilitated crossing of the aortic arch and positioning. The self-alignment after release of the stabilization arches makes the ACURATE neo particularly suitable for horizontal aortic configurations.

\section{Access route}

The access route is a key determinant of device selection. Currently, there are only two devices that can be used for both transapical and transvascular access: the SAPIEN 3/Ultra and the ACURATE neo. The most important criterion for the transvascular access is the diameter of the sheath or the delivery system (in sheathless devices) that determine the choice of the prosthesis. The ratio between the size of the sheath and the vessel diameter has been described as a predictor of vascular complications (50). In the event of very small vessel diameters $(<5 \mathrm{~mm})$, the Evolut R may be most suitable given its very low profile. Alternatively, the sheathless insertion of the Portico system can be taken into consideration (51). It should be noted that the sheath sizes declared by most manufacturers represent inner diameters, and maximum outer diameters can differ considerably, especially upon insertion of the delivery system (also see Table 1).

Mild to moderate tortuosity of the iliac arteries or the aorta usually straightens upon insertion of a stiff wire or large-bore sheath, whereas severe kinking can indeed be challenging, especially in combination with circular calcification and/or narrowing of the vessel lumen. Very rarely, in tall subjects with tortuous vessels, delivery systems may be too short. Contrary to the widespread misconception, the presence of an abdominal aortic aneurysm does not preclude transfemoral access, as the aneurysm is typically completely protected by the introducer sheath.

\section{Coexisting morbidities and potential complications}

\section{Conduction disturbances}

Left bundle branch block and conduction disturbances with requirement for PPI belong to the most frequent complications encountered after TAVR. The presence of a right bundle branch block at baseline is known as an independent patient-related predictor of the need for PPI. Several other risk factors have been described, including the presence of a porcelain aorta, the absence of prior valve surgery, septal hypertrophy, calcification of the left- or non-coronary cusp, prosthesis depth in the left ventricular outflow tract, calcification of the device landing zone, and larger or significantly oversized prostheses $(52,53)$. The reported rate of PPI varies considerably as a function of the type of THV (Table 2). The selection of the appropriate THV should be made according to this knowledge and the perceived individual patient-related risk factors for PPI. A recent publication by Jilaihawi et al. focused on an individualized approach where the implantation depth of the Medtronic Evolut R/PRO was adapted to the length of the membranous septum measured in computed tomography (54). As a result, the rates of new PPI and new left bundle branch block were significantly reduced with this approach compared with the rated for standard implantation techniques (54).

\section{Coronary artery disease}

The impact of coronary artery disease on procedural and long-term outcome after TAVR is yet unclear. Most institutions pursue a strategy of complete revascularization prior to TAVR. Indeed, incomplete revascularization was demonstrated to be an independent predictor of decreased left ventricular recovery and was associated with higher 1 -year mortality (55). In contrast, complete coronary revascularization was not a prerequisite in elderly patients prior to transcatheter aortic valve implantation (TAVI), 


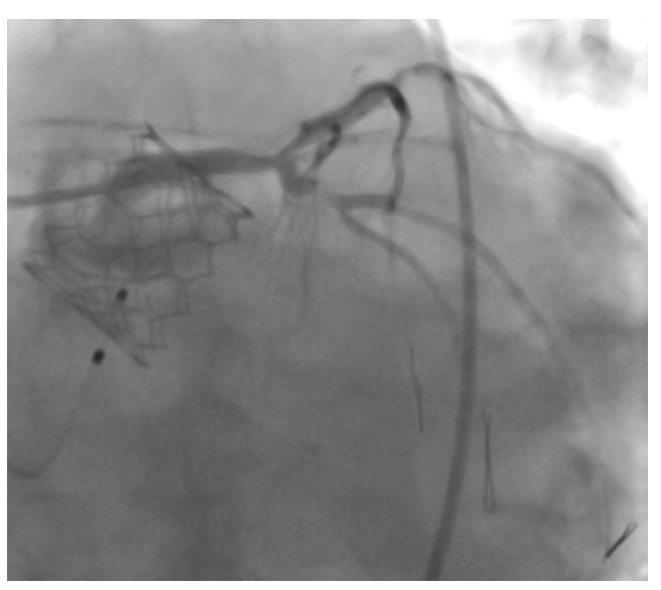

Figure 2 Even complex coronary interventions are possible after TAVR, as illustrated by the case of this 93 -year-old male patient. He had undergone implantation of a SAPIEN 3 valve and then developed cardiogenic shock. Unrestricted access permitted percutaneous coronary intervention with good outcome. TAVR, transcatheter aortic valve replacement.

provided the revascularization strategy was selected by a dedicated heart team (56). Subject to the extent of coronary artery disease and anticipated future need for revascularization after TAVR, it should be considered that access to the coronary ostia can be challenging with certain types of THV in situ. However, the SAPIEN 3 and SAPIEN 3 Ultra typically permit unrestricted future reaccess to the coronary arteries (Figure 2).

\section{Prosthesis-patient mismatch}

Hemodynamic characteristics of the different THVs vary mainly as a function of leaflet position. Supra-annular prostheses have been shown to have lower transvalvular gradients, and particularly in patients with small aortic root dimensions, the incidence of patient-prosthesis mismatch is lower for supra-annular THVs (57). In the clinical routine, this particular aspect gets less attention than it deserves, since patient-prosthesis mismatch affects long-term outcome (58).

\section{Reduced left ventricular function}

Patients with reduced left ventricular function and severe aortic stenosis have a poorer prognosis after aortic valve replacement due to the associated pathological irreversible myocardial fibrosis. In the absence of reliable evidence, it can only be anticipated that rapid pacing, conduction abnormalities, relevant PVL, and high post-procedural gradients are particularly detrimental in cases of severely reduced left ventricular function. In the event that after TAVR a patient becomes pacemaker-dependent with the need for persistent right ventricular pacing, the implantation of a cardiac resynchronization therapy system should be considered.

\section{Renal failure}

The amount of contrast material required for a standard TAVR procedure differs between b-exp and s-exp devices. In view of recent results showing significantly reduced contrast usage for implantation of the b-exp prosthesis SAPIEN 3 in direct comparison with s-exp devices, the selection of a b-exp prosthesis is the best choice, based on current knowledge (50). Alternatively, an approach employing little or even no contrast material may be attempted (59).

\section{Miscellaneous factors}

\section{Ease of use}

The various principles of deployment and features such as resheathability or retrievability illustrate the complexity of procedural handling of modern TAVR devices. Even though "ease of use" as a parameter is difficult to measure, experienced operators will agree that usability of the available THV systems varies and will impact the learning curve. The ease of use is a rather underestimated aspect that affects procedural safety and success. Thus, the risk of human error or technical failure increases with the complexity of the procedural steps.

\section{Radiation dose}

Due to the various underlying principles of contemporary THVs, there are also differences regarding the radiation burden experienced by both patients and operators. Higher radiation doses can be anticipated with increasing duration and complexity of the procedure. This pertains to the need for pre- or post-dilatation and to devices with complex positioning or maneuvers like resheathing or retrieval of the device. Whereas long-term effects of radiation exposure may not be relevant in elderly patients, this aspect has to be considered when indications are expanded to younger populations or those with lower risk.

\section{Durability}

As comparative data on long-term performance of the currently available THV systems are lacking, valve durability cannot yet be used as a criterion for differential selection. 


\section{Conclusions}

None of the currently available THVs are capable of addressing the full spectrum of challenging patient anatomies and clinical situations in a comprehensive fashion. Theoretically, such a device should have a small profile for vascular access, allow straightforward and safe application, deliver good immediate and long-term results in terms of PVL and hemodynamics, be suitable for a large variety of aortic root anatomies including severe calcification or bicuspid aortic valves, and have a low PPI rate. However, some devices may be more appropriate for use in specific patients and result in better outcomes than others. The question of which or how many different devices a center should keep in stock mainly depends on the TAVR volume. Centers with low volumes usually have only one or few valve types in stock, including a THV system that covers the entire annulus range. In centers with higher volumes, it is recommended to have different devices that are complementary in terms of radial force and annulus range. Due to the specific advantages and disadvantages of each THV system, it is strongly advisable to make use of the broad spectrum of available treatment options in TAVR technology and to select the most appropriate device according to the individual anatomy and comorbidities of the patient.

\section{Acknowledgments}

We thank Elizabeth Martinson, PhD, of the KHFI Editorial Office for excellent editorial work.

Funding: None.

\section{Footnote}

Provenance and Peer Review: This article was commissioned by the Guest Editor (Daniel Hernández-Vaquero) for the series"Structural Heart Disease: The Revolution"published in Annals of Translational Medicine. The article was sent for external peer review organized by the Guest Editor and the editorial office.

Conflicts of Interest: The series "Structural Heart Disease: The Revolution" was commissioned by the editorial office without any funding or sponsorship. WK received speaker fees from Abbott Vascular, Boston Scientific, Edwards Lifesciences, Medtronic. MR received speaker fees from Abbott Vascular.
Ethical Statement: The authors are accountable for all aspects of the work in ensuring that questions related to the accuracy or integrity of any part of the work are appropriately investigated and resolved.

Open Access Statement: This is an Open Access article distributed in accordance with the Creative Commons Attribution-NonCommercial-NoDerivs 4.0 International License (CC BY-NC-ND 4.0), which permits the noncommercial replication and distribution of the article with the strict proviso that no changes or edits are made and the original work is properly cited (including links to both the formal publication through the relevant DOI and the license). See: https://creativecommons.org/licenses/by-nc-nd/4.0/.

\section{References}

1. Baumgartner H, Falk V, Bax JJ, et al. 2017 ESC/EACTS Guidelines for the management of valvular heart disease. Eur Heart J 2017;38:2739-91.

2. Nishimura RA, Otto CM, Bonow RO, et al. 2017 AHA/ ACC Focused Update of the 2014 AHA/ACC Guideline for the Management of Patients With Valvular Heart Disease: A Report of the American College of Cardiology/ American Heart Association Task Force on Clinical Practice Guidelines. J Am Coll Cardiol 2017;70:252-89.

3. Thyregod HG, Steinbrüchel DA, Ihlemann N, et al. Transcatheter Versus Surgical Aortic Valve Replacement in Patients With Severe Aortic Valve Stenosis: 1-Year Results From the All-Comers NOTION Randomized Clinical Trial. J Am Coll Cardiol 2015;65:2184-94.

4. Leon MB, Smith CR, Mack MJ, et al. Transcatheter or Surgical Aortic-Valve Replacement in Intermediate-Risk Patients. N Engl J Med 2016;374:1609-20.

5. Reardon MJ, Van Mieghem NM, Popma JJ, et al. Surgical or Transcatheter Aortic-Valve Replacement in IntermediateRisk Patients. N Engl J Med 2017;376:1321-31.

6. Popma JJ, Deeb GM, Yakubov SJ, et al. Transcatheter Aortic-Valve Replacement with a Self-Expanding Valve in Low-Risk Patients. N Engl J Med 2019;380:1706-15.

7. Mack MJ, Leon MB, Thourani VH, et al. Transcatheter Aortic-Valve Replacement with a Balloon-Expandable Valve in Low-Risk Patients. N Engl J Med 2019;380:1695-705.

8. Webb J, Gerosa G, Lefèvre T, et al. Multicenter evaluation of a next-generation balloon-expandable transcatheter aortic valve. J Am Coll Cardiol 2014;64:2235-43.

9. Kodali S, Thourani VH, White J, et al. Early clinical and echocardiographic outcomes after SAPIEN 3 transcatheter 
aortic valve replacement in inoperable, high-risk and intermediate-risk patients with aortic stenosis. Eur Heart J 2016;37:2252-62.

10. Wendler O, Schymik G, Treede H, et al. SOURCE 3 Registry: Design and 30-Day Results of the European Postapproval Registry of the Latest Generation of the SAPIEN 3 Transcatheter Heart Valve. Circulation 2017;135:1123-32.

11. Saia F, Gandolfo C, Palmerini T, et al. Sapien 3 Ultra balloon-expandable transcatheter aortic valve: in-hospital and 30-day results from the multicentre S3U registry. EuroIntervention 2019. [Epub ahead of print].

12. Manoharan G, Walton AS, Brecker SJ, et al. Treatment of Symptomatic Severe Aortic Stenosis With a Novel Resheathable Supra-Annular Self-Expanding Transcatheter Aortic Valve System. JACC Cardiovasc Interv 2015;8:1359-67.

13. Grube E, Van Mieghem NM, Bleiziffer S, et al. Clinical Outcomes With a Repositionable Self-Expanding Transcatheter Aortic Valve Prosthesis: The International FORWARD Study. J Am Coll Cardiol 2017;70:845-53.

14. Forrest JK, Mangi AA, Popma JJ, et al. Early Outcomes With the Evolut PRO Repositionable Self-Expanding Transcatheter Aortic Valve With Pericardial Wrap. JACC Cardiovasc Interv 2018;11:160-8.

15. Möllmann H, Walther T, Siqueira D, et al. Transfemoral TAVI using the self-expanding ACURATE neo prosthesis: one-year outcomes of the multicentre "CE-approval cohort". EuroIntervention 2017;13:e1040-6.

16. Kim WK, Hengstenberg C, Hilker M, et al. The SAVI-TF Registry: 1-Year Outcomes of the European Post-Market Registry Using the ACURATE neo Transcatheter Heart Valve Under Real-World Conditions in 1,000 Patients. JACC Cardiovasc Interv 2018;11:1368-74.

17. Meredith IT, Walters DL, Dumonteil N, et al. 1-Year Outcomes With the Fully Repositionable and Retrievable Lotus Transcatheter Aortic Replacement Valve in 120 High-Risk Surgical Patients With Severe Aortic Stenosis: Results of the REPRISE II Study. JACC Cardiovasc Interv 2016;9:376-84.

18. Montone RA, Testa L, Fraccaro C, et al. Procedural and 30-day clinical outcomes following transcatheter aortic valve replacement with lotus valve: Results of the RELEVANT study. Catheter Cardiovasc Interv 2017;90:1206-11.

19. Falk V, Wöhrle J, Hildick-Smith D, et al. Safety and efficacy of a repositionable and fully retrievable aortic valve used in routine clinical practice: the RESPOND Study.
Eur Heart J 2017;38:3359-66.

20. Götberg M. One-year outcomes with the transcatheter LOTUS Edge Aortic Valve System. PCR London Valves; 2018; London, United Kingdom.

21. Willson AB, Rodès-Cabau J, Wood DA, et al. Transcatheter aortic valve replacement with the St. Jude Medical Portico valve: first-in-human experience. J Am Coll Cardiol 2012;60:581-6.

22. Manoharan G, Linke A, Moellmann H, et al. Multicentre clinical study evaluating a novel resheathable annular functioning self-expanding transcatheter aortic valve system: safety and performance results at 30 days with the Portico system. EuroIntervention 2016;12:768-74.

23. Maisano F, Worthley S, Rodés-Cabau J, et al. Early commercial experience from transcatheter aortic valve implantation using the Portico ${ }^{\mathrm{TM}}$ bioprosthetic valve: 30 day outcomes in the multicentre PORTICO-1 study. EuroIntervention 2018;14:886-93.

24. Fontana GP. Portico Re-sheathable Transcatheter Aortic Valve System US IDE Trial. San Francisco, CA, USA. Transcatheter Cardiovascular Therapeutics; 2019.

25. Wenaweser P, Stortecky S, Schütz T, et al. Transcatheter aortic valve implantation with the NVT Allegra transcatheter heart valve system: first-in-human experience with a novel self-expanding transcatheter heart valve. EuroIntervention 2016;12:71-7.

26. Jagielak D, Stanska A, Klapkowski A, et al. Transfermoral aortic valve implantation using self-expanding New Valve Technology (NVT) Allegra bioprosthesis: A pilot prospective study. Cardiol J 2019. [Epub ahead of print].

27. Sharma SK, Rao RS, Chandra P, et al. First-in-Human Evaluation of Balloon Expandable Transcatheter Heart Valve in the Treatment of Severe Symptomatic Native Aortic Stenosis: The MyVal-1 Study. EuroIntervention 2019. [Epub ahead of print].

28. Cribier A, Eltchaninoff H, Bash A, et al. Percutaneous transcatheter implantation of an aortic valve prosthesis for calcific aortic stenosis: first human case description. Circulation 2002;106:3006-8.

29. Husser O, Pellegrini C, Kessler T, et al. Predictors of Permanent Pacemaker Implantations and New-Onset Conduction Abnormalities With the SAPIEN 3 BalloonExpandable Transcatheter Heart Valve. JACC Cardiovasc Interv 2016;9:244-54.

30. Barbanti M, Yang TH, Rodès Cabau J, et al. Anatomical and procedural features associated with aortic root rupture during balloon-expandable transcatheter aortic valve replacement. Circulation 2013;128:244-53. 
31. Hellhammer K, Piayda K, Afzal S, et al. The Latest Evolution of the Medtronic CoreValve System in the Era of Transcatheter Aortic Valve Replacement: Matched Comparison of the Evolut PRO and Evolut R. JACC Cardiovasc Interv 2018;11:2314-22.

32. Ojeda S, Hidalgo F, Romero M, et al. Impact of the repositionable Evolut R CoreValve system on the need for a permanent pacemaker after transcatheter aortic valve implantation in patients with severe aortic stenosis. Catheter Cardiovasc Interv 2020;95:783-90.

33. Kim WK, Mollmann H, Liebetrau C, et al. The ACURATE neo Transcatheter Heart Valve: A Comprehensive Analysis of Predictors of Procedural Outcome. JACC Cardiovasc Interv 2018;11:1721-9.

34. Toggweiler S, Nissen H, Mogensen B, et al. Very low pacemaker rate following ACURATE neo transcatheter heart valve implantation. EuroIntervention 2017;13:1273-80.

35. Schäfer U, Butter C, Landt M, et al. Thirty-day outcomes of a novel transcatheter heart valve to treat degenerated surgical valves: the VIVALL multicentre, single-arm, pilot study. EuroIntervention 2019;15:e757-63.

36. Sathananthan J, Sellers S, Barlow A, et al. Overexpansion of the SAPIEN 3 Transcatheter Heart Valve: An Ex Vivo Bench Study. JACC Cardiovasc Interv 2018;11:1696-705.

37. Shivaraju A, Kodali S, Thilo C, et al. Overexpansion of the SAPIEN 3 Transcatheter Heart Valve: A Feasibility Study. JACC Cardiovasc Interv 2015;8:2041-3.

38. Herrmann HC, Daneshvar SA, Fonarow GC, et al. Prosthesis-Patient Mismatch in Patients Undergoing Transcatheter Aortic Valve Replacement: From the STS/ ACC TVT Registry. J Am Coll Cardiol 2018;72:2701-11.

39. Walther T, Hamm CW, Schuler G, et al. Perioperative Results and Complications in 15,964 Transcatheter Aortic Valve Replacements: Prospective Data From the GARY Registry. J Am Coll Cardiol 2015;65:2173-80.

40. Eggebrecht H, Mehta RH. Transcatheter aortic valve implantation (TAVI) in Germany 2008-2014: on its way to standard therapy for aortic valve stenosis in the elderly? EuroIntervention 2016;11:1029-33.

41. Harrison JK, Hughes GC, Reardon MJ, et al. Balloon Post-Dilation Following Implantation of a Self-Expanding Transcatheter Aortic Valve Bioprosthesis. JACC Cardiovasc Interv 2017;10:168-75.

42. Généreux P, Head SJ, Van Mieghem NM, et al. Clinical outcomes after transcatheter aortic valve replacement using valve academic research consortium definitions: a weighted meta-analysis of 3,519 patients from 16 studies. J
Am Coll Cardiol 2012;59:2317-26.

43. Ribeiro HB, Webb JG, Makkar RR, et al. Predictive factors, management, and clinical outcomes of coronary obstruction following transcatheter aortic valve implantation: insights from a large multicenter registry. J Am Coll Cardiol 2013;62:1552-62.

44. Fonseca P, Figueiredo B, Almeida C, et al. Aortic Valve Calcium Volume Predicts Paravalvular Regurgitation and the Need for Balloon Post-Dilatation After Transcatheter Aortic Valve Implantation. J Interv Cardiol 2016;29:117-23.

45. Philip F, Faza NN, Schoenhagen P, et al. Aortic annulus and root characteristics in severe aortic stenosis due to bicuspid aortic valve and tricuspid aortic valves: implications for transcatheter aortic valve therapies. Catheter Cardiovasc Interv 2015;86:E88-98.

46. Kim WK, Gaede L, Husser O, et al. Computed Tomography for Diagnosis and Classification of Bicuspid Aortic Valve Disease in Transcatheter Aortic Valve Replacement. JACC Cardiovasc Imaging 2018;11:1539-40.

47. Makkar RR, Yoon SH, Leon MB, et al. Association Between Transcatheter Aortic Valve Replacement for Bicuspid vs Tricuspid Aortic Stenosis and Mortality or Stroke. JAMA 2019;321:2193-202.

48. Yoon SH, Lefèvre T, Ahn JM, et al. Transcatheter Aortic Valve Replacement With Early- and New-Generation Devices in Bicuspid Aortic Valve Stenosis. J Am Coll Cardiol 2016;68:1195-205.

49. Yoon SH, Bleiziffer S, De Backer O, et al. Outcomes in Transcatheter Aortic Valve Replacement for Bicuspid Versus Tricuspid Aortic Valve Stenosis. J Am Coll Cardiol 2017;69:2579-89.

50. Lanz J, Kim WK, Walther T, et al. Safety and efficacy of a self-expanding versus a balloon-expandable bioprosthesis for transcatheter aortic valve replacement in patients with symptomatic severe aortic stenosis: a randomised noninferiority trial. Lancet 2019;394:1619-28.

51. Taramasso M, Denegri A, Kuwata S, et al. Feasibility and safety of transfemoral sheathless portico aortic valve implantation: Preliminary results in a single center experience. Catheter Cardiovasc Interv 2018;91:533-9.

52. Khatri PJ, Webb JG, Rodés-Cabau J, et al. Adverse effects associated with transcatheter aortic valve implantation: a meta-analysis of contemporary studies. Ann Intern Med 2013;158:35-46.

53. Barbanti M, Gulino S, Costa G, et al. Pathophysiology, incidence and predictors of conduction disturbances during Transcatheter Aortic Valve Implantation. Expert Rev Med Devices 2017;14:135-47. 
54. Jilaihawi H, Zhao Z, Du R, et al. Minimizing Permanent Pacemaker Following Repositionable Self-Expanding Transcatheter Aortic Valve Replacement. JACC Cardiovasc Interv 2019;12:1796-807.

55. Freixa X, Chan J, Bonan R, et al. Impact of coronary artery disease on left ventricular ejection fraction recovery following transcatheter aortic valve implantation. Catheter Cardiovasc Interv 2015;85:450-8.

56. Van Mieghem NM, van der Boon RM, Faqiri E, et al. Complete revascularization is not a prerequisite for success in current transcatheter aortic valve implantation practice. JACC Cardiovasc Interv 2013;6:867-75.

57. Miyasaka M, Tada N, Taguri M, et al. Incidence,

Cite this article as: Renker M, Kim WK. Choice of transcatheter heart valve: should we select the device according to each patient's characteristics or should it be "one valve fits all”? Ann Transl Med 2020;8(15):961. doi: 10.21037/ atm.2020.04.13
Predictors, and Clinical Impact of Prosthesis-Patient Mismatch Following Transcatheter Aortic Valve Replacement in Asian Patients: The OCEAN-TAVI Registry. JACC Cardiovasc Interv 2018;11:771-80.

58. Pibarot P, Weissman NJ, Stewart WJ, et al. Incidence and sequelae of prosthesis-patient mismatch in transcatheter versus surgical valve replacement in high-risk patients with severe aortic stenosis: a PARTNER trial cohort--a analysis. J Am Coll Cardiol 2014;64:1323-34.

59. Castriota F, Nerla R, Micari A, et al. Contrast-Zero Transcatheter Aortic Valve Replacement for Patients With Severe Renal Dysfunction: A Single-Center Experience. JACC Cardiovasc Interv 2018;11:820-2. 